\title{
MT3FT-150R0204122: Report on the acquisition and installation of FEI Talos F200X S/TEM
}

\author{
Chad M. Parish \\ Materials Science and Technology Division, Oak Ridge National Laboratory \\ Oak Ridge, TN, USA. Email: parishcm@ornl.gov
}

\begin{abstract}
Objective
This document describes the installation, performance, and early results from the FCRD-funded acquisition of a new advanced analytical scanning / transmission electron microscopy (S/TEM) at the Oak Ridge National Laboratory Low Activation Materials Development and Analysis (LAMDA) facility.
\end{abstract}

\section{Description of the instrument}

The advanced analytical S/TEM instrument purchased and installed is the FEI company "Talos" F200X. This tool was configured to optimize it for metallurgy and ceramics studies, such as those required by accident-tolerant fuel concepts ( $\mathrm{FeCrAl}, \mathrm{SiC}$, etc.)

The instrument is a $200 \mathrm{keV} \mathrm{S/TEM,} \mathrm{equipped} \mathrm{with} \mathrm{a} \mathrm{proprietary} \mathrm{"XFEG"} \mathrm{high-brightness} \mathrm{Schottky-}$ emission field-emission-gun electron source, which provides beams $\sim 5 \times$ brighter than previous Schottky-FEG technologies. The objective lens is a constant-power "ATWIN" asymmetric analytical objective lens. The combination of high-brightness illumination from the XFEG and the constantpower objective lens provides an excellent resolution specification of $0.16 \mathrm{~nm}$ in STEM mode. The asymmetric objective lens also houses a proprietary "SuperX" energy-dispersive spectroscopy Xray detector system, which consists of four silicon-drift-detector (SDD) units, arranged symmetrically around the sample (Figure 1a). This provides 0.9 srad solid angle of collection for Xrays, compared to $\sim 0.06$ srad in the previously-used ORNL analytical S/TEM, a Philips CM200STFEG (vintage 1994). Combining the high-brightness source and high-collection efficiency SDD system, along with modern electronics, computers, and pulse processors, allows factors of 50-100× improvements in X-ray spectrum imaging efficiency over the CM200 system. This will provide major savings in time and cost for experiments on the metal and ceramic samples examined by the FCRD projects at ORNL LAMDA.

The instrument is housed in an enclosure to reduce environmental disturbances, and has been placed in the LAMDA lab's microscopy suite. This allows shirt-sleeve (rather than contaminationzone clothing) operation and service of the instrument, but also allows irradiated materials (including alpha-bearing materials, with special work controls) to be examined. This is illustrated in Figure 1b.

In addition to the SuperX system, additional detectors include four STEM detectors (high-angle annular dark field, medium-angle annular dark field, low angle annular dark field, and bright field) 
and a high-speed, high-resolution (4096×4096 pixel) TEM camera system. This is a generalpurpose, non-specialized configuration ideally suited for metallurgy and ceramics studies.
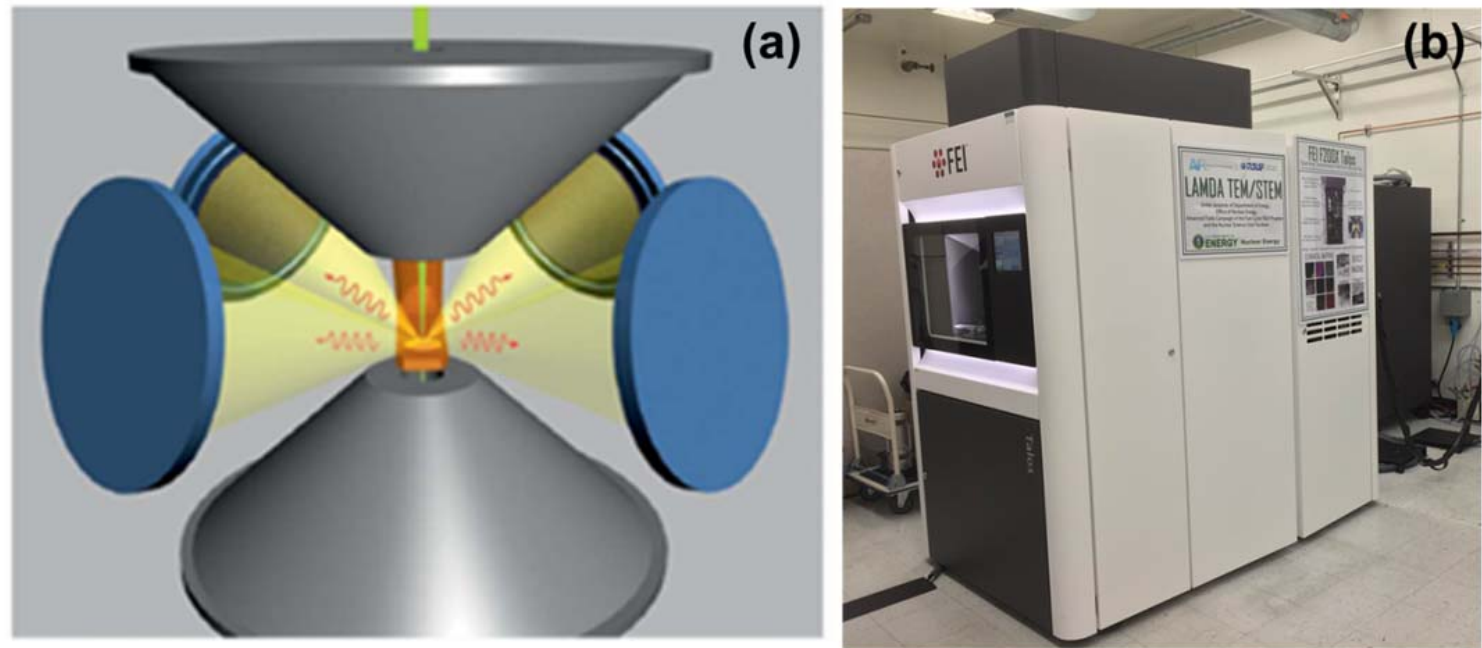

Figure 1: (a) schematic illustration of SuperX 4-sector detector. Image courtesy FEI company. (b) The ORNL FCRD Talos in position.

\section{Site acceptance testing}

The instrument was placed in the laboratory (ORNL Building 4508, Room 136) on 31 March, 2015. Utilities (208 VAC single-phase power with uninterruptible power supply and mains-matching stepup autotransformer, cooling water, and compressed air) were installed, and power was applied 12 May, 2015, followed by NRTL safety inspection, then conditioning of the high voltage and ultrahigh vacuum. The first beam was obtained 2 June 2015, and the instrument was accepted and began imaging irradiated samples 9 July 2015.

The instrument passed all necessary site-acceptance tests for performance and stability. The TEM imaging information limit exceeds 120 pm (Figure 2a-2b), as measured using evaporated Au-Pd droplets on thin carbon. The TEM camera is $4096 \times 4096$ pixel, which allows high-resolution images to be acquired of large areas, illustrated in Figure 2c. 


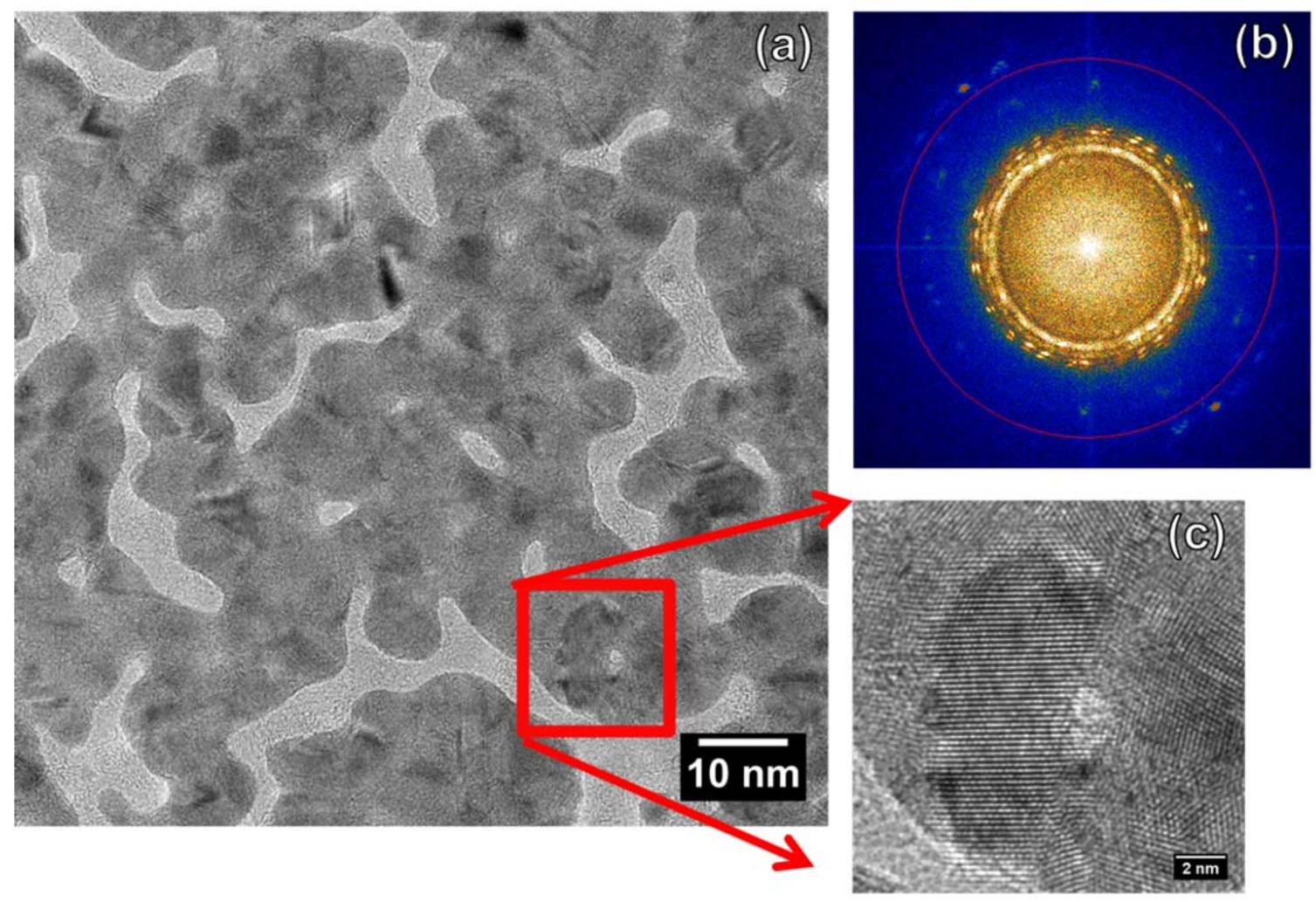

Figure 2: TEM information limit testing. (a) Image of AuPd droplets. (b) Fourier transform. Red circle is 120 pm; clear diffractogram peaks are seen outside this limit. (c) Enlarged region of the figure in (a), illustrating the capabilities of the $4096 \times 4096$ pixel camera.

The STEM image resolution exceeds $160 \mathrm{pm}$ (Figure 3a-3c), as illustrated on the $<101>$ zone axis of NITE SiC accident-tolerant-fuel material, where $\{202\}_{\beta \text {-SiC }}$ reflections (known spacing $154 \mathrm{pm}$ ) are observed.
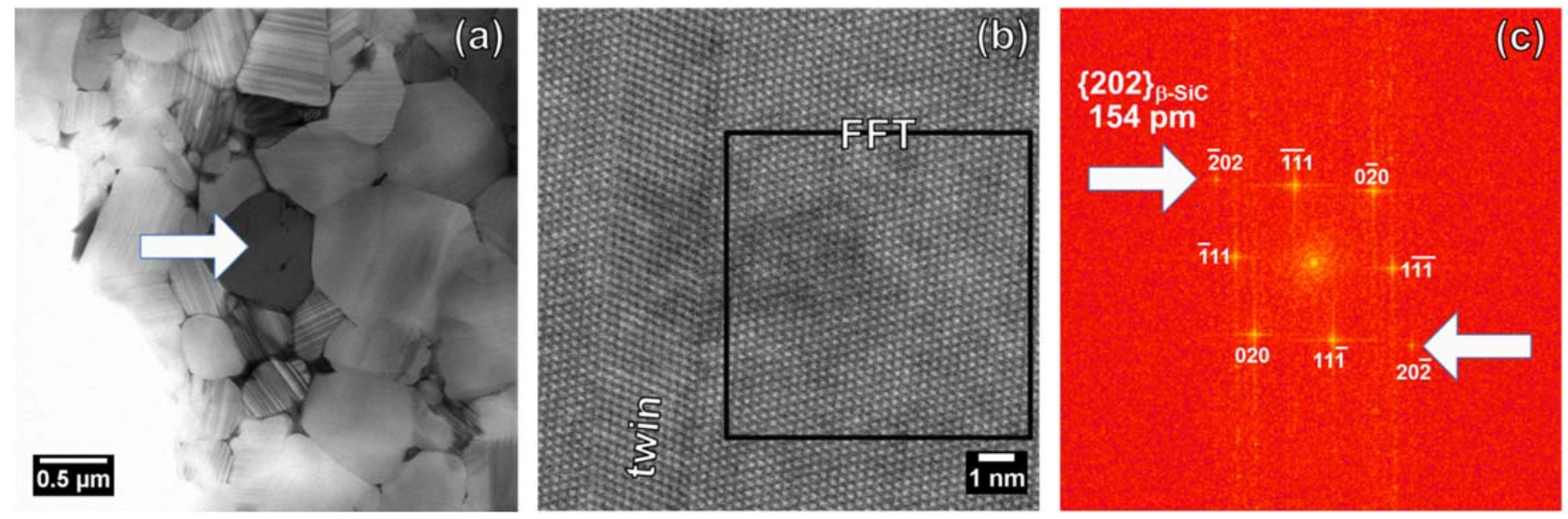

Figure 3: (a) Grain structure of NITE-SiC. Dark grain, arrowed, is tilted to [101] zone. (b) Highmagnification image of the marked grain. (c) Fourier transform of the boxed area in (b), with the 154 pm reflections of $\{202\}_{\beta \text {-SiC }}$ marked. 
Additional site-acceptance tests, such as probe current, X-ray leakage, and X-ray spectroscopy energy resolution, were also passed.

\section{Preliminary results}

Preliminary results exceeded all expectations. Three examples will be given, one for neutronirradiated FeCrAl accident-tolerant-fuel candidate alloy*, one for oxide-dispersion strengthened (ODS) FeCrAl†, and one for NITE silicon carbideł.

The FeCrAl alloy invesitagated is Alkorthal 720 with a nominal composition of Fe-13Cr-4Al+minor solute additions (in wt.\%). This alloy was, irradiated at $\sim 382^{\circ} \mathrm{C}$ to $1.8 \mathrm{dpa}$ in the High Flux Isotope Reactor and hence was an activated sample. A random high angle grain boundary is tilted edge-on, Figure $4 \mathrm{a}$, and dislocations are visible. (The tilt angles were very large, but because of the symmetry of the SuperX system, two of the four SDD sectors were illuminated by the specimen, allowing highquality data to be obtained despite the high tilt angles, which is a capability only this FEI system has). X-ray maps (Figure 4b-e) and linescan data (Figure 4f) are extracted from the spectrum image dataset. $\mathrm{Cr}, \mathrm{Al}$, and $\mathrm{Si}$ radiation-induced segregation are visible, and in the grains, $\mathrm{Cr}$-rich pockets

( $\alpha^{\prime}$ phase) are visible. This is possibly the first observation of $\alpha^{\prime}$ phase using S/TEM techniques, rather than the much more time-consuming energy-filtered TEM or atom-probe methods for $\alpha^{\prime}$ identification. A color overlay of the $\mathrm{Fe}, \mathrm{Cr}$, and $\mathrm{Al}$ maps (Figure 4g) shows the radiation-induced segregation and the $\alpha^{\prime}$ phase (green) more clearly.

* FeCrAl data is courtesy Dr. Kevin G. Field, ORNL.

† Sample courtesy Drs. David Hoelzer and Sebastien Dryepondt, ORNL.

‡ Sample courtesy Drs. Kurt Terrani and Ritesh Sachan, ORNL. 

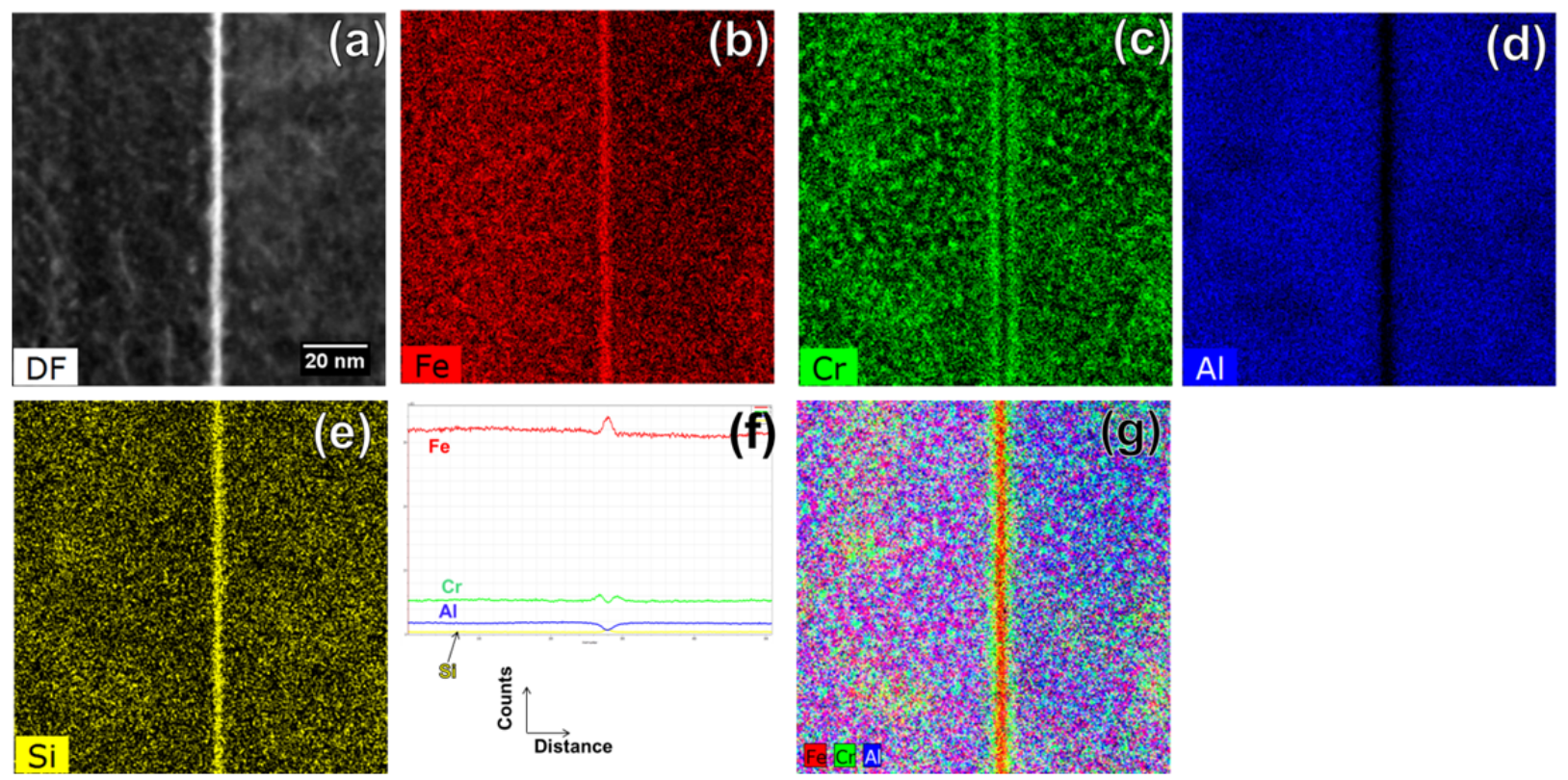

Figure 4: (a) dark field STEM image and (b-e) X-ray maps of neutron-irradiated FeCrAl. (f) X-ray count profile. (g) Color overlay showing segregation and Cr-rich $\alpha^{\prime}$ formation. Map time: 14 minutes. $3 x 3$ pixel averaging kernel.

The ODS FeCrAl sample shows a high density of very small oxide particles, Figure 5. This dataset illustrates two other advantages of the Talos instrument, namely, very-high-resolution mapping $(\sim$ $500 \mathrm{pm} / \mathrm{pix}$ in this case) and high sensitivity to low energy X-rays, specifically 0 in the oxide particles and $\mathrm{C}$ in the carbide phases. Complex multiphase precipitates are identified (center).
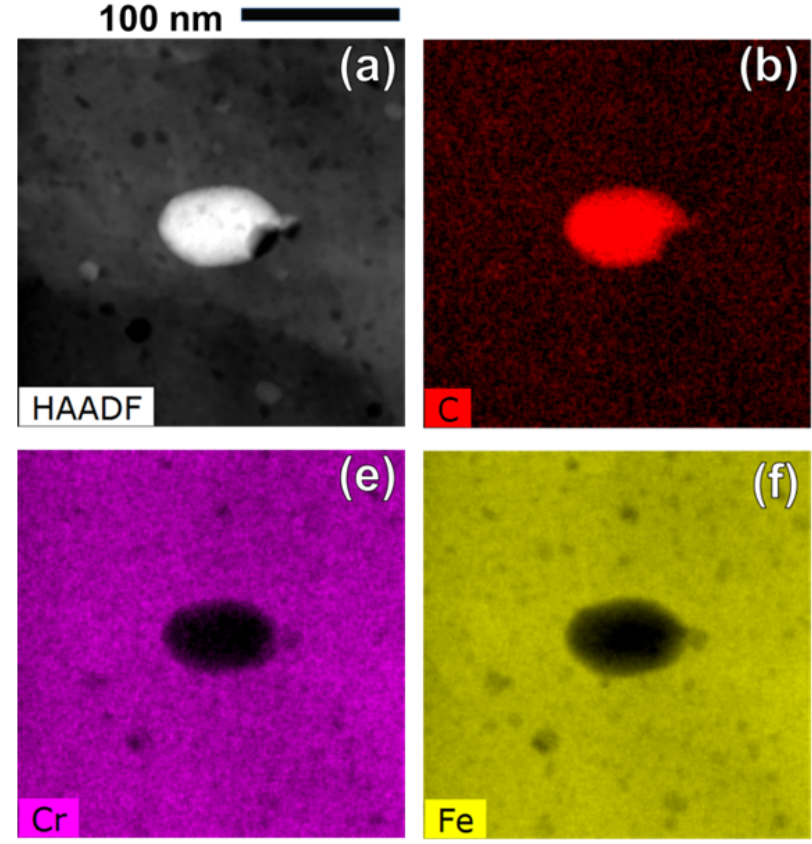
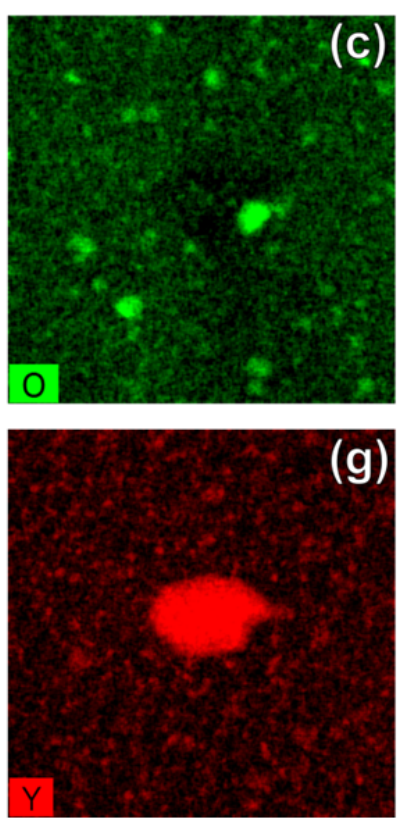
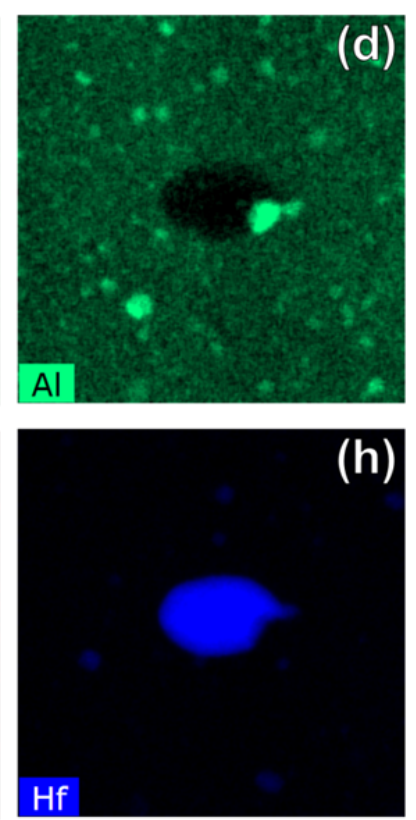

Figure 5: (a) HAADF image and (b-h) X-ray maps of a Y-Zr FeCrAl alloy. Map time: 16 minutes. 
NITE SiC contains oxide phases in the carbide matrix, and its analysis benefits from the Talos instrument, also. In particular, the carbide and oxide phases can be damaged by high-dose highenergy electrons, so the efficient X-ray collection allows maps to be obtained with minimal damage. An oxide packet at a triple-point, with oxide extending down the grain boundaries, is imaged in TEM mode in Figure 6. X-ray spectrum imaging in STEM mode shows that the oxides are inhomogeneous, with different grain boundaries being wet by different cations (Al, Y, Zr, Ce), Figure 7. For instance, Ce appears to surround the $\mathrm{Zr}$ at the triple-junctions (arrows) and to alone wet the vertical grain boundary at the bottom of the frame.

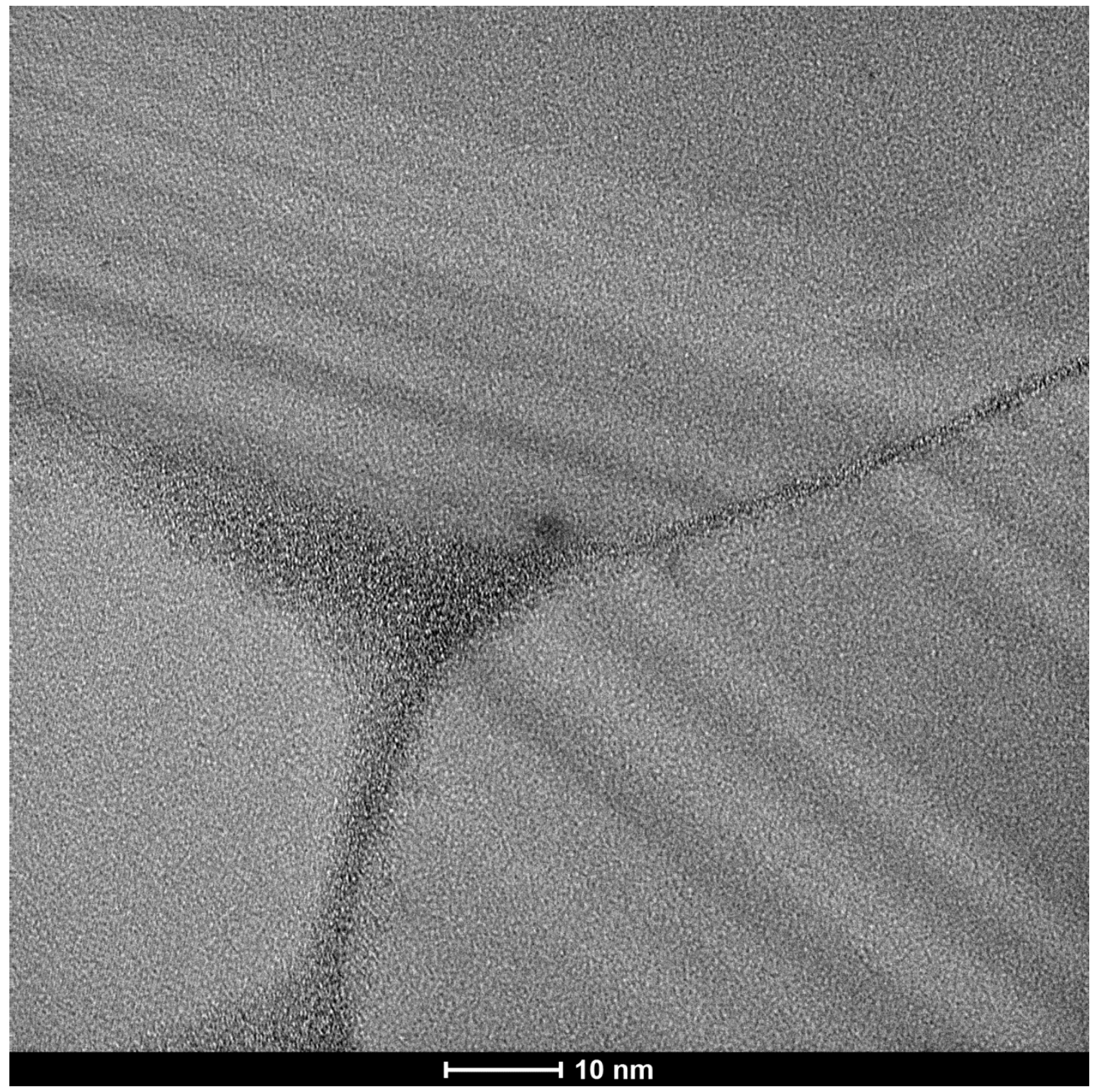

Figure 6: High-resolution TEM image of oxides near a grain triple-junctions in NITE-SiC. 

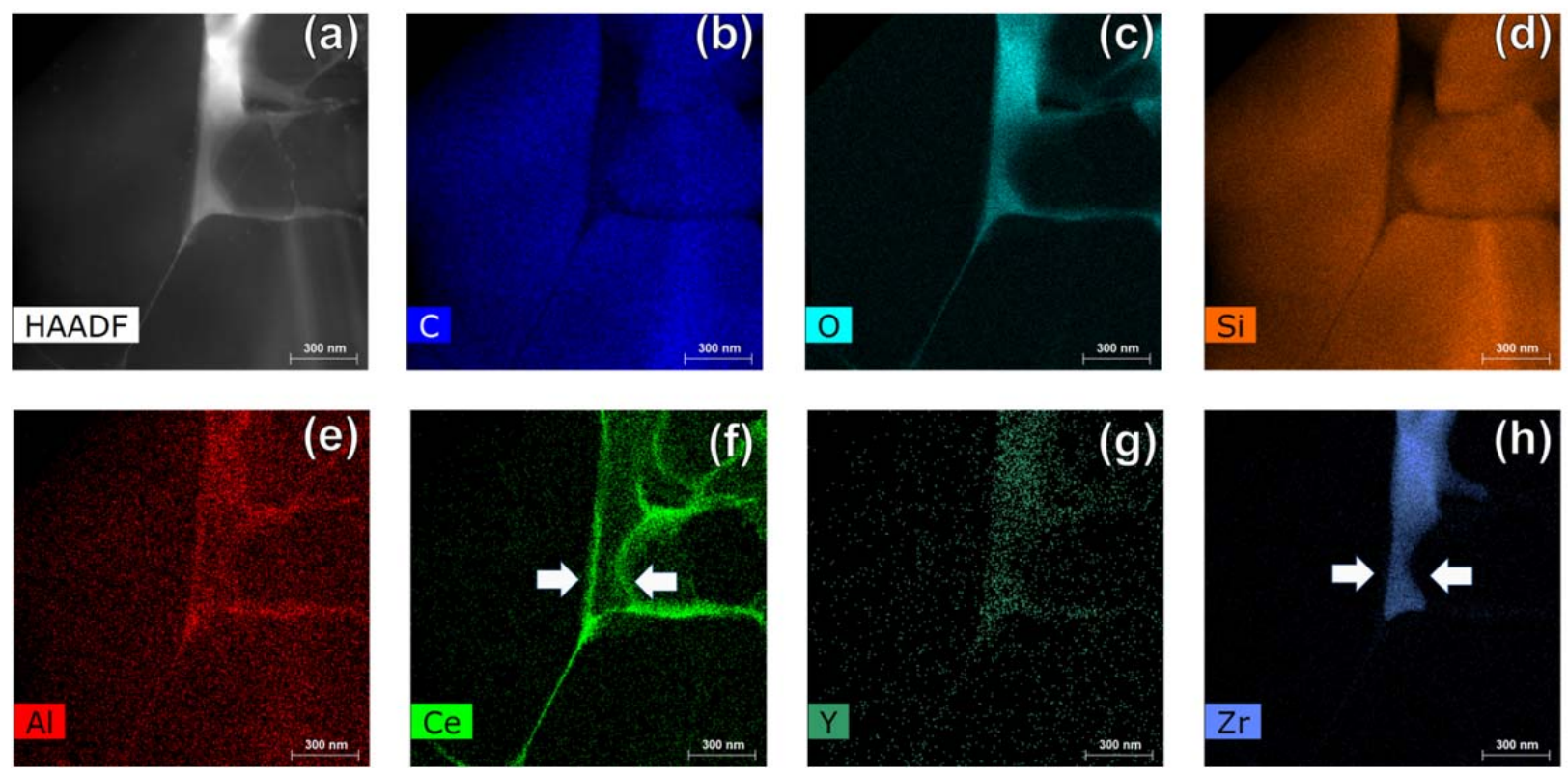

Figure 7: (a) HAADF image and (b-h) X-ray maps of NITE SiC. Map time: 349 sec.

\section{Conclusions}

The FEI F200X Talos advanced analytical S/TEM instrument has been successfully installed and put into operation at ORNL LAMDA lab. The instrument is intended primarily to study irradiated structural nuclear materials and fuels, and the instrument is already in heavy use to support Nuclear Energy and FCRD projects, and exciting results are expected in the future. 